\title{
Atmospheric Shocks in Mira Variables - MgII Emission
}

\author{
Edward W. Brugel \\ Center for Astrophysics and Space Astronomy \\ University of Colorado \\ Boulder, CO 80309 (USA) \\ T. E. Beach, L.A. Willson and G. H. Bowen \\ Physics Department \\ Iowa State University \\ Ames, Iowa 50011 (USA)
}

\begin{abstract}
We have undertaken an extensive program to investigate atmospheric shock dynamics in Mira variables through monitoring the MgII emission strengths versus phase. Theoretical calculations of Bowen (1987), which represent the structural variations in the extended atmosphere of a pulsating Mira, are presented in addition to the MgII observations. The model calculations generate density, temperature and velocity structures as determined by stellar parameters and by interactions of the driving amplitude, dust opacity and thermal relaxation processes. The main diagnostic provided by the models is the total emitted shock luminosity versus phase, which can be compared to the observed $\mathrm{MgII}$ emission. Models and observations are presented for three Miras: S Car, R Car and T Cep.
\end{abstract}

\section{OBSERVATIONS AND DYNAMIC ATMOSPHERE MODELS}

The LWP camera on the International Ultraviolet Explorer (IUE) has been used to monitor several Mira variables. The essential ultraviolet data acquired through this long-term monitoring program are the flux in the $\mathrm{h}$ and $\mathrm{k}$ lines of MgII. In addition, the IUE FESmagnitudes are used to ascertain an approximate visual magnitude and corresponding phase of the visible light curve.

Dynamic atmosphere models for comparison with the MgII luminosity data were generated using a code by Bowen (1987). The code emphasizes the modeling of the dynamic nature of the atmospheres, and utilizes simplified radiative transfer - a grey spherical atmosphere with constant opacity. Although detailed light curves are not generated by the models, the actual light curves can be interpreted in terms of the shock structure shown. The peak in the MgII luminosity of a Mira typically occurs between phase 0.2 and 0.4 of the visual light curve, and can be interpreted as occuring when the main shock reaches an optical depth of $\tau(2800 \AA)=2 / 3$. 


\section{COMPARISON OF MODELS TO MGII DATA}

Model atmospheres were prepared for comparison with S Car, R Car, and T Cep using the parameters listed in Table 1. All models assume fundamental mode pulsation. Three driving amplitudes were used for each model: Model $1 \mathrm{a}, 1 \mathrm{~b}$, and $1 \mathrm{c}$ were driven at 1.5, 2.5, and $3.5 \mathrm{~km} / \mathrm{s}$, respectively. Models 2 and 3 used 2, 3 , and $4 \mathrm{~km} / \mathrm{s}$ for $\mathrm{a}, \mathrm{b}$, and $\mathrm{c}$.

Table 1

Model Parameters

\begin{tabular}{|lcccc|}
\hline & Mass $\left(\mathcal{M}_{\odot}\right)$ & $\mathrm{T}_{\text {eff }}(\mathrm{K})$ & Radius $\left(\mathcal{R}_{\odot}\right)$ & Period (days) \\
\hline Model 1 / S Car & 0.8 & 3143 & 150 & 149 \\
Model 2 / R Car & 1.2 & 3067 & 255 & 308 \\
Model 3 / T Cep & 1.4 & 3030 & 305 & 387 \\
\hline
\end{tabular}

The models give us the total post shock luminosity $L_{\text {shock }}$ as a function of phase, but only a fraction of this luminosity is from MgII. To compare the model with the data we would need to know the ratio $L_{M g I} / L_{\text {shock }}$ as a function of phase. Unfortunately, this ratio is a function of temperature, density, and thermodynamic history of the post-shock gas, and is not easily calculated for the non-LTE conditions in the post-shock region. When fitting the data to the model curves, priority was given to matching the data points for which the shock was at low optical depths, since this region of the model suffers least from the simplified radiative transfer calculations used.

The best correspondence between models and data was found using the parameters listed in Table 2. A range of acceptable fits can be found near these "best fits" by using a proportional increase or decrease of both the opacity and luminosity ratios.

A good correspondence could not be found between the S Car data and Model 1 at any of the driving amplitudes. This lack of agreement between model and data may be the result of using an unsuitable model (perhaps S Car pulsates in an overtone mode); or the simplifications used are most inaccurate for the shorter period pulsators.

Table 2

Model Parameters For "Best" Data Fits

\begin{tabular}{|lcccc|}
\hline & $\begin{array}{c}\text { Driving } \\
\text { amplitude } \\
(\mathrm{km} / \mathrm{s})\end{array}$ & $\begin{array}{c}\kappa(2800 \AA) / \\
\kappa(\text { Rosseland })\end{array}$ & $\begin{array}{c}L_{M g I I} / \\
L_{\text {shock }}\end{array}$ & $\begin{array}{c}\Delta \phi \\
\text { (light phase - } \\
\text { driving phase) }\end{array}$ \\
\hline T Cep/Model 3c & 4 & 1000 & 0.009 & 0.075 \\
T Cep/Model 3b & 3 & 2000 & 0.060 & 0.075 \\
R Car/Model 2c & 4 & 1000 & 0.070 & 0.100 \\
S Car/Model 1 & - & - & - & - \\
\hline
\end{tabular}

\section{REFERENCES}

Bowen, G.H. 1987, Ap.J. (submitted). 